DOE/ID/13765

Pointwise Energy Solution of the Boltzmann Transport Equation for Thermal Neutrons

Final Report - 07/01/1999 - 06/30/2001

M. L. Williams

June 2001

Work Performed Under Contract No. DE-FG07-99ID13765

For

U.S. Department of Energy

Assistant Secretary for

Energy Efficiency and Renewable Energy

Washington, DC

By

Louisiana State University

New Orleans, LA 
DOE/ID/13765

\title{
POINTWISE ENERGY SOLUTION OF THE BOLTZMANN TRANSPORT EQUATION FOR THERMAL NEUTRONS \\ FINAL REPORT \\ 07/01/1999 - 06/30/2001
}

\author{
Mark L. Williams
}

June 2001

Work Performed Under Contract No. DE-FG07-99ID13765

\author{
Prepared for the \\ U.S. Department of Energy \\ Assistant Secretary for \\ Energy Efficiency and Renewable Energy \\ Washington, DC
}

\author{
Prepared by \\ Louisiana State University \\ New Orleans, LA
}




\author{
FINAL REPORT for \\ D.O.E. Nuclear Engineering Education Research Program \\ Grant No. DE-FG07-99ID13765 \\ POINTWISE ENERGY SOLUTION OF THE \\ BOLTZMANN TRANSPORT EQUATION FOR THERMAL NEUTRONS \\ Mark L Williams \\ Louisiana State University \\ Nuclear Science Center/ Dept of Physics \\ June 2001
}

\title{
SUMMARY
}

In July of 1999 Louisiana State University (LSU) was awarded a two year research grant by the D.O.E. NEER program to develop a methodology for neutron transport calculations using pointwise (PW) nuclear data in the thermal energy range, and to implement the method into the CENTRM transport code being developed at LSU for Oak Ridge National Laboratory (ORNL). This work has extended CENTRM's current epithermal PW calculation to encompass the thermal range, providing a continuous-energy deterministic transport code that can address problems that may not be adequately treated using multigroup methods.

The new version of the CENTRM code was completed, and provided to ORNL for inclusion in the next release of the SCALE code system. The new thermal calculation developed by the NEER project is a significant improvement in the CENTRM capability, and should have an impact on criticality and shipping cask analysis done by numerous organizations who use this code system.. It has already been utilized by several ORNL staff for criticality safety analysis cases, and results have been presented by them at several conferences.

The new version of CENTRM has been verified by comparing computed eigenvalues for pincell geometries to results obtained by Monte Carlo and other established codes. Good agreement was obtained. 


\section{Introduction}

For several years a one-dimensional, continuous-energy neutron transport code has been under development by Louisiana State University (LSU), in cooperation with Oak Ridge National Laboratory. The code--- named "CENTRM for Continuous ENergy TRansport Module--- computes spacedependent angular and/or scalar fluxes and Legendre moments, using a continuous energy representation over the energy range usually required for reactor physics and criticality safety calculations ( 0-20 MeV). CENTRM uses a combination of multigroup (MG) and pointwise (PW) discrete ordinates calculations to solve the Boltzmann equation. Infinite homogeneous media and onedimensional slab, spherical, or cylindrical geometries can be modeled in CENTRM, with general boundary conditions, and with arbitrary spatial meshing, temperature distributions, and directional quadrature. The Legendre scattering order is arbitrary for the MG portion of the calculations, but the PW solution is usually limited to P5 for heavy materials (A>100) and P7 for lighter moderators.

CENTRM typically performs a PW calculation within the resolved resonance range of most fuel materials. PW nuclear data for CENTRM are processed from basic ENDF/B data, while MG data is taken from available AMPX formatted libraries. Within the upper and lower MG ranges, the MG flux solution is divided by the group lethargy width to obtain average flux per lethargy values, assigned to the mid-lethargy of the group; while fluxes computed within the PW range correspond to the flux per lethargy at the discrete points of the PW energy mesh. The PW calculation provides very high energy resolution of flux spectra with fine-structure, while the $\mathrm{MG}$ calculations produce more slowly varying spectra characteristic of "smooth" cross sections. A continuous-energy spectrum for the fluxes and flux moments, as a function of space and angle (or Legendre moment), is represented by linear variation between the computed flux per lethargy values.

The neutron flux represented on such an ultra-fine energy mesh is able resolve spectral finestructure caused by resonance self-shielding and cross-shielding. Because the flux spectrum is calculated as a function of space, the effects of variations in material composition and temperature are treated realistically. Continuous-energy scalar fluxes and Legendre moments computed by CENTRM are well suited for use as weighting functions to generate problem-dependent MG cross sections. For this reason CENTRM is being incorporated as a resoance processing module in the SCALE system of 
nuclear analysis codes developed by ORNL. An auxiliary code named PMC (Prepare Multigroup Cross sections) has been developed by LSU to use the computed PW fluxes to process self-shielded MG data within the resolved resonance range, for subsequent MG calculations with higher dimensional transport codes, such as the Monte Carlo code KENO. Another function may be to perform cell burnup calculations with the ORIGEN depletion code in SCALE, in which CENTRM would generate problem-dependant MG cross sections for determining reaction rates in fuel and fission product nuclides.

One of the primary limitations in the original version of CENTRM is that scattering within the PW range is assumed to be elastic scatter from stationary nuclei. The assumption of a stationary nucleus is reasonable for the energy range above $\sim 3 \mathrm{eV}$. However below this energy range, the impact of nuclear motion is no longer negligible. In the thermal range, nuclear motion causes upscattering reactions to occur; and molecular bonding and coherent scatter may be significant for several important moderators such as light or heavy water, and graphite. The PW calculation method now in CENTRM does not treat thermal scattering effects correctly. This is the reason that the current CENTRM algorithm must switch from a PW to a MG solution within the lower MG range. Unfortunately the current approach precludes a PW calculation for the thermal flux spectra, which could be used to generate problem-dependent group cross sections for materials such as ${ }^{239} \mathrm{Pu},{ }^{240} \mathrm{Pu}$, and ${ }^{241} \mathrm{Pu}$ that have important thermal resonances.

Self-shielding of thermal resonances may have a significant impact on criticality and reactivity coefficients. Currently available methods for self-shielding MG cross sections in the thermal range do not account for thermal scattering effects (viz, neutron upscattering into resonances), nor do they consider the spatial variation of resonance self-shielding, which depends on temperature gradients (because of Doppler broadening). Overlapping of Pu resonances with those of absorber and fission product nuclides-- such as Sm-149, Eu-151, Rh-103, Kr-83, Hf-177, and others -- results in crossshielding effects not treated in conventional resonance shielding methods. Furthermore, the large thermal absorption cross sections of some nuclides, such as lumped burnable poisons and control rod materials, cause severe spatial gradients in the thermal flux at material interfaces, and distort the thermal spectrum from Maxwellian behavior, which is usually assumed in processing MG thermal cross sections. These effects impact the MG data for the thermal energy range. There are no cross section processing 
codes available to generate problem-specific MG data, taking into account thermal scattering reactions, self-shielding, resonance overlap effects, and spatial variations in the temperature distribution and flux spectrum. This requires a PW transport calculation for the thermal energy range.

The purpose of the NEER project was to develop a methodology for neutron transport calculations using PW nuclear data in the thermal energy range, and to implement the method into the CENTRM transport code. The methodology was to be general in its ability to treat arbitrary spacedependent material composition and temperature distributions, anisotropic scatter, free-gas as well as bound moderators, and incoherent as well as coherent thermal scattering reactions.

\section{Results}

Table 1. shows the milestones for the two-year NEER grant. All of the milestones were completed within the available budget and time period, with the exception of the "heavy gas" model implementation.. We found that good accuracy was obtained using the standard free-gas scatter kernel, with requiring the heavy-gas approximation. Although preliminary coding was included in CENTRM to use the heavy gas model, it has not been fully implemented at this time, since it will not be used for standard calculations.

A significant amount of time was required to determine the best overall approach for treating free-gas kernels as well as bound scattering using $\mathrm{S}(\mathrm{a}, \mathrm{B})$ data. For the latter, a method based on the modified FLANGE approach proposed by Robinson was used to computed Legendre moments of the free-gas kernel. This was found to run very fast and give accurate results even for heavy materials. Legendre moments for incoherent-inelastic and coherent-elastic scatter kernels of bound materials were computed in CENTRM by interpolation of pre-calculated PW moments stored in a external data file. These data were processed from ENDF/B data $S(a, B)$ using a new ORNL code. It was found necessary to use a variation of the "unit-base transform" interpolation approach to get accurate kernels. The final methodology was verified to give extremely reliable kernel moments.

Since the ultimate goal of this work was to develop a practical program that could be used for applications addressed by the SCALE code system, efficient running time was a primary concern for the pointwise (PW) thermal calculation. Outer iterations of the PW thermal solution were accelerated by using a MG thermal calculations to obtain an initial guess for the thermal spctrum, and by using new 
routines for space-dependant rebalance of the integrated thermal band flux. We were able to construct numerial algorithms that appear to work very well even for energy meshes consisting of $~ 30,000$ total points and $\sim 1000$ points in the thermal range. Typical pincell cases take about 5-10 minutes to execute on a SUN Workstation.

Appendix A summarizes the new methodology developed for the CNETRM PW thermal calculation.

\section{Impact of NEER Support}

The NEER project at LSU provided support for one graduate student at LSU in Nuclear Engineering, who worked on the project as part of his thesis research. At this time the student is still at LSU working on his graduate degree. The funding also provided support for a post-graduate research associate in nuclear engineering, as well as partial summer support for one nuclear engineering faculty.

However the major impact of the NEER support was development an improved version of CENTRM, the first deterministic program capable of performing PW thermal neutron calculations. The new version of CENTRM was provided to ORNL for inclusion in the next release of the SCALE code system. The new thermal calculation developed by the NEER project is a significant improvement in the CENTRM capability, and should have an impact on criticality and shipping cask analysis done by numerous organizations who use this code system.. It has already been utilized by several ORNL staff for criticality safety analysis cases, and results have been presented by them at several conferences.

Code documentation was written as part of this project, and has been provided to ORNL to be published as part of the next release of SCALE report. A draft journal article has also been completed, and will be submitted by the end of this calendar year. 


\title{
Table 1. \\ Milestones for NEER Project
}

\begin{abstract}
$\underline{\underline{\text { First Year Milestones }}}$
1. Develop required numerical expressions for PW scatter source moments;

2. Modify current PW calculation to perform outer iterations in thermal range

3. Implement PW thermal calculation for free-gas materials and Heavy Gas Model
\end{abstract}

\section{$\underline{\underline{\text { Second year Milestones }}}$}

4. Implement PW thermal scatter for bound inelastic incoherent, $\mathrm{S}(\mathrm{a}, \boldsymbol{B})$ data

5. Verifiy PW thermal calculations by comparison with MCNP results

6. Perform benchmark calculations for LWR and MOX fuel pins 


\section{APPENDIX A. \\ Description of CENTRM Thermal Methodology \\ Developed for DOE NEER Project}

\section{INTRODUCTION}

A computational method based on the discrete ordinates approach was recently developed to solve the one-dimensional Boltzmann transport equation using pointwise (PW) cross section data. (REF). This method has been implemented in a program called CENTRM (REF), which will be included in the next release of the SCALE code system developed by Oak Ridge National Laboratory for criticality safety and shipping cask analysis. (REF) CENTRM computes space-dependent, continuous-energy neutron spectra, that may be used to process resonance-shielded multigroup (MG) data for subsequent analysis with Monte Carlo (KENO or MORSE codes)(REF) and deterministic methods.

In the original CENTRM development, PW Legendre moments of the neutron scattering source were evaluated using a scattering kernel based on elastic, s-wave neutron interactions with stationary nuclei. (REF) This assumption is generally adequate for treating epithermal reactions of most reactor fuel nuclides, which typically have a resolved resonance range below about $10 \mathrm{keV}$. The method was subsequently extended to treat discrete-level inelastic reactions as well, so that resonance shielding of most structural materials could also be treated. (REF) However, the code neglected effects of molecular motion on scattering kinematics (other than its impact on Doppler broadening); thus the PW transport calculation could not be extended into the thermal energy range. This work describes implementation and testing of a PW discrete ordinates method for thermal neutron transport calculations with CENTRM. Together with the earlier developments, the current calculation algorithm provides capability for a PW discrete ordinates solution over the full energy range of interest for most neutron transport applications.

A PW discrete ordinates calculation is an effective approach to obtain problem-specific thermal neutron spectra for MG data processing. In current MG processing the spectral weight function in the thermal range is usually assumed to be a simple Maxwellian distribution, sometimes divided by the macroscopic cross section to approximate resonance self-shielding effects. In highly absorbing regions, 
such as reactor fuel pins, lumped burnable poisons, and control rods, the neutron spectrum may deviate considerably from Maxwellian. Also, there are several important nuclides,-- including ${ }^{239} \mathrm{Pu}$, ${ }^{240} \mathrm{Pu}$, and several fission products-- that have large resonances in the thermal energy range, so that thermal upscattering may affect the degree of resonance self-shielding. It has been shown that epithermal MG cross sections of resonance nuclides can change drastically as a function of position inside of a fuel pin, due to variations in resonance self-shielding. (REF) Similar variations in thermal MG data may also occur near the fuel-clad-moderator interfaces where the thermal spectrum changes with space. The large temperature gradients also may impact the thermal neutron distribution, and possibly reactivity temperature coefficients and burnable poison worth. 


\section{METHODOLOGY FOR PW THERMAL CALCULATION}

Transport calculations in CENTRM generally use a combination of MG and PW techniques, requiring PW data from a CENTRM Cross Section Library, and MG data from a standard AMPX Working Library, respectively.(REF) Typically, a MG transport calculation is done within the highest range of energy, called the "Upper MG Range (UMR)", where cross section data tend to be smoother, and scattering more anisotropic. This is followed by a PW calculation within the resonance range, and then optionally another MG calculation in the "Lower MG Range (LMR)". Cutoffs between the various ranges are specified by input.

A continuous spectrum is obtained by linear representation of the flux per lethargy, between values computed at points in the solution lethargy mesh (in MG ranges, the flux per lethargy is assigned to the mid-point lethargy of a group). Scattering from one range to another is computed from appropriate cross section and kinematics data, along with MG and/or PW flux moments obtained in the discrete ordinates solution, so that a consistent set of continuous-spectrum scalar fluxes and flux moments are obtained throughout the entire space-energy domain.

Prior to this work, it was required in CENTRM that the thermal energy range be contained within the LMR, but this restriction has now been removed. For example, it is possible to perform PW calculations over part of the thermal range, and MG for the remainder. If desired, a PW calculation can be performed over essentially the full energy range. However, even if a full PW calculation is done, a MG nuclear data library is still required for CENTRM, because the group structure must be known for subsequent MG data processing operations. Also, MG data are used optionally to represent cross sections of materials not contained in the PW data library, and MG calculations are used for iterationacceleration operations discussed in II.B.

\section{II.A. Energy Mesh Determination}

The lethargy mesh used for the PW flux calculation in the thermal range is determined in a similar manner to that for the epithermal range. A set of union energy meshes is first generated, by combining energy meshes of the total cross sections for all nuclides in each respective mixture. Energy meshes of individual nuclides include all important structure (eg, resonances, Bragg edges, etc.) contained in the PW cross sections generated by the POLIDENT (REF) code directly from the basic ENDF/B data files. A PW macroscopic total cross section is computed for each mixture, at every point in its union 
mesh; and then the energy meshes of the respective mixtures are thinned, based on an input-specified error tolerance for linear interpolation of cross sections between adjacent points. The flux solution mesh is obtained by combining all thinned energy meshes for every mixture, adding points for the MG boundaries, and converting into lethargy for the actual flux calculation. The lethargy mesh is finally checked to insure that it meets certain minimum mesh criteria for accurate computation of the scattering source. In the thermal range these criteria are as follows:

$$
\begin{array}{ll}
\left.0.1 \mathrm{eV}<\mathrm{E}<\mathrm{E}_{\mathrm{TH}}\right): & \text { maximum } ? \mathrm{u}<0.005 \\
0.0 \mathrm{eV}<\mathrm{E}<0.1 \mathrm{eV}: & \text { maximum } ? \mathrm{u}<0.01
\end{array}
$$

If necessary, additional points are added to satisfy the above constraints. For comparison, the maximum lethargy mesh size in the epi-thermal range for typical reactor fuel material is constrained to be $? \mathrm{u}<0.003$. A typical thermal lethargy mesh for low enriched uranium fuels consists $\sim 1500$ points, in contrast to the epithermal mesh that may contain $20,000-30,000$ points in order to adequately represent resolved resoance data. Because the lethargy mesh is defined sufficiently fine that linear flux variation can be assumed between points, the efficient trapezoidal method can be used for evaluating lethargy-integrated quantities.

\section{II.B. Thermal Flux Solution}

The PW thermal solution first begins with a MG thermal calculation, consisting of several outer iterations $(\sim 10)$ to obtain a flux guess. The computed MG flux moments at all space intervals are multiplied by MG macroscopic cross sections to determine the MG collision density, which is generally a more slowly varying function of lethargy than the flux. The MG collision density moments per lethargy are mapped onto the flux lethargy mesh, and divided by PW macroscopic cross sections to get an initial estimate of PW flux moments for determining the thermal scattering source. Subsequent values of the PW flux moments are computed by solving the PW transport equation using additional outer iterations.

Within a given outer iteration, the PW angular flux per unit lethargy in the thermal range is

$$
\Omega \bullet \Delta \Psi\left(\mathrm{r}, \mathrm{u}_{\mathrm{n}}, \Omega\right)+\Sigma_{\mathrm{t}}\left(\mathrm{r}, \mathrm{u}_{\mathrm{n}}\right) \Psi=\sum_{\ell \mathrm{k}=1}^{\mathrm{LK}} \frac{2 \ell+1}{2} \mathrm{Y}_{\ell \mathrm{k}}(\Omega) \mathrm{Q}_{\ell \mathrm{k}}\left(\mathrm{r}, \mathrm{u}_{\mathrm{n}}\right)+\mathrm{Q}_{\mathrm{ext}}\left(\mathrm{r}, \mathrm{u}_{\mathrm{n}}, \Omega\right)
$$

calculated from the neutron transport equation evaluated at lethargy mesh point "n", The last term on the right side of eq. (1) is the external source for the thermal range, including fixed 
sources and down-scatter from the epithermal range. The latter is computed from a submoment expansion, in the same manner as points in the epithermal range. The first term on the right side is the

scattering source due to thermal neutrons, expressed in terms of the "LK" scattering source moments, The above summation is over all nuclides " $\mathrm{j}$ " at the respective spatial location, and the number (LK) of source moments is a function of Legendre expansion order and geometry type.

As in the epithermal calculation, CENTRM uses the discrete ordinates approach to solve eq. (1) at all lethargy points in the thermal range. However there are significant differences in epithermal and thermal solutions. In the epithermal range, neutrons can only lose energy in scattering reactions, so that a single sweep from high to low energy (IE, low to high lethargy) is required in the solution. Since low energy neutrons may gain as well as lose energy in scattering reactions, outer iterations are required to converge the thermal scattering source. Furthermore, the PW scatter kernels $S_{1}\left(u^{\prime} \rightarrow u\right)$ in the epithermal range are assumed to represent two-body interactions (such as elastic and discrete-level inelastic reactions) between a neutron and a stationary nucleus, with scattering distributions isotropic in the center of mass coordinate system. The simple kinematic relations allow the efficient "sub-moment expansion" and "cumulative integral" method to be utilized in computing the scattering source moments. (REF) Thermal scattering reactions are not two body reactions, but rather represent an effective average over the molecular velocity distribution; thus, there is no simple kinematic relationship between neutron energy loss and the angle of scatter relative to its initial direction. For these reasons the thermal scatter source must be determined in a different manner from that described in reference 1.

In the numerical solution for thermal neutrons, the lethargy integral in eq. (2) for the thermal-range scatter source at lethargy $\mathrm{u}_{\mathrm{n}}$ is approximated as a summation over all " $\mathrm{N}$ " points in the

thermal range,

where $\mathrm{m}=1$ is the thermal/epithermal boundary point; $\mathrm{m}=\mathrm{N}$ is the lowest energy point; and $A_{m}$ are standard quadrature weights for trapezoidal integration with $\mathrm{N}-1$ lethargy panels: 


$$
\begin{aligned}
& A_{m}=0.5 \times\left(? u_{m}+? u_{m+1}\right) ; \quad \text { for } m=2,3, \ldots N-1 \\
& 0.5 \times ? \mathrm{u}_{\mathrm{m}} \quad ; \quad \text { for } \mathrm{m}=1 \text { or } \mathrm{N}
\end{aligned}
$$

For a given outer iteration, the scattering source at lethargy $u_{n}$ is evaluated from eqs. (2) and (3) using the most recently computed values for the flux moments. Since the solution mesh is swept from high to low energy, this means that flux moments corresponding to $\mathrm{m}<\mathrm{n}$ in eq. (3) are calculated using angular fluxes from the current outer iteration, while those for $m>n$ are from the previous outer. Due to the presence of PW flux moments at lethargy point $\mathrm{m}=\mathrm{n}$ in eq. (3), inner iterations are necessary to converge the "with-in point" (no energy loss) contribution of the thermal scattering source in eq. (1). As discussed in ref. (1), no inner iterations are required to converge the with-in point source term in epithermal PW calculation, because there is no change in the neutron direction if there is no energy loss. (REF)

A space-dependent rebalance calculation for the entire thermal energy band is performed between outer iterations in order to speed up convergence of the PW discrete ordinates solution. (REF) Reaction rates and leakage values appearing in the thermal-band rebalance equation are obtained by integrating PW (and/or MG) values over the thermal range. Other acceleration techniques, such as over-relaxation and renormalization, are also employed.

\section{COMPUTATION OF THE THERMAL SCATTERING KERNELS}

Since thermal neutrons have energies comparable to the mean kinetic energy of molecules in thermal equilibrium, the scattering kernels must account for molecular motion. If internal atomic bonding effects are neglected, so that atoms of a material act independently like a gas at the temperature of the medium, then the analytical free-gas kernel can utilized for the differential scattering function.(REF) CENTRM uses the free gas model for all materials except for a relatively few important moderator materials for which thermal scattering laws are available in the ENDF/B nuclear data files. Scattering law data account for effects of molecular bonding and possibly polyatomic crystalline structure. While free-gas kernels are calculated internally, the kernel moments for bound thermal scatterers have been pre-processed from ENDF/B, and stored in a data file that is accessed by CENTRM. Treatment of the kernels for free-gas and bound scatterers are described in more detail in the following two sections.

\section{III.A. Free Gas Kernels}


CENTRM computes free gas kernels using the approach proposed by Robinson (REF) as a modification to the original FLANGE (REF) methodology. Legendre moments of the free-gas kernel,

$$
\sigma_{\ell}\left(\mathrm{u}^{\prime} \rightarrow \mathrm{u}\right)=\mathrm{A} \sigma_{\mathrm{b}} \frac{\mathrm{E}}{\mathrm{E}^{\prime}} \mathrm{e}^{-\beta / 2} \sum_{\mathrm{n}=0}^{\ell} \mathrm{W}_{\ell \mathrm{n}} \mathrm{H}_{\mathrm{n}}\left(\mathrm{E}^{\prime}, \mathrm{E}\right)
$$

per unit lethargy, are expressed as,

where $W_{l n}$ are constants, $s$ free is the constant free-atom cross section and $H_{n}$ are a-moments of the free-

gas scatter law, given as (see definition of a and B parameters in "Notation" section)

The limits on the above integral correspond to:

$$
\begin{gathered}
a_{L}\left(E^{\prime}, E\right)=a\left(E^{\prime}, E, \mu_{0}=-1\right) ; \text { and } a_{H}\left(E^{\prime}, E\right)=a\left(E^{\prime}, E, \mu_{0}=1\right) . \\
H_{n}\left(E^{\prime}, E\right)=2(2 n-1) H_{n-1}+\beta^{2} H_{n-2}-\left[F_{n}\left(\sqrt{\alpha_{H}}, \beta\right)-F_{n}\left(\sqrt{\alpha_{L}}, \beta\right)\right]
\end{gathered}
$$

The alpha moments $\mathrm{H}_{n}$ can be evaluated very efficiently using a recursive relation (REF):

$$
F_{n}(t, \beta)=\frac{t^{2 n-1} e^{\frac{1}{4}\left(\frac{\beta^{2}}{t^{2}}+t^{2}\right)}}{\sqrt{\pi} / 2}
$$

where $F_{n}$ is the function,

Analytical expressions for the initial two moments, $\mathrm{H}_{0}$ and $\mathrm{H}_{1}$, are given in reference ??? REF

The free-gas kernel is based on the assumption of a constant thermal scattering cross section; therefore, to approximately account for energy dependence of the thermal cross section (eg, due to low energy resonances), the moments are multiplied by the ratio $s \mathrm{~s}(\mathrm{E}) / \mathrm{s}_{\mathrm{FG}}(\mathrm{E})$, where ss is the Doppler broadened scatter cross section processed from $\mathrm{ENDF} / \mathrm{B}$ data, and $\mathrm{s}_{\mathrm{FG}}$ is the free-gas scatter cross section obtained by integrating eq. (4) for $1=0$ over all final lethargy values. The integration can be done analytically to obtain,(REF) 


$$
\sigma\left(E^{\prime}\right)=\frac{\sigma_{\text {free }}}{y^{2}}\left[\left(y^{2}+\% o\right) \operatorname{erf}(y)+\frac{y^{-y^{2}}}{\sqrt{\pi}}\right]
$$

where 